\title{
V wie Versicherungsmedizin oder das stimmige Bild
}

\section{Dominik Heim}

PD Dr. med., Facharzt für Chirurgie, Mitglied FMH

Es gibt in letzter Zeit wieder jene kleinen, stillen Filme, bei denen man beim Abspann - ganz am Ende, wenn die ersten Kinozuschauer schon längst aus ihren tiefen Plüschsesseln aufstehen - eigentlich noch gerne sitzen bleiben und weiterschauen würde. Die Rede ist nicht von der nächsten Folge von Game of Thrones oder von Netflix-Serien. Wobei, aufgepasst mit der kulturellen Überheblichkeit: Das diesjährige Filmfestival in Venedig hat - im Gegensatz zu den Filmfestspielen in Cannes - diesem im Internet abrufbaren Sender keine Absage erteilt und erntete dafür Szenen-Anerkennung. Und der Goldene Löwe ging denn auch prompt an Roma/Netflix! Bei einigen dieser Filme aber von Stille zu sprechen, würde einer neuen Bedeutung dieses
Wortes gleichkommen. Nein, die Rede ist von Filmen, in denen scheinbar unbedeutende, weil alltägliche $\mathrm{Ge}$ schichten erzählt werden:

Vielleicht ein Liebesfilm wie The Guernsey Literary and Potato Peel Pie Society. Er spielt in der Zeit der deutschen Besetzung der gleichnamigen Insel und vor allem danach. Das erwartet glückliche Ende will fast nicht kommen, immer wieder wird mit der Geduld des Zuschauers gespielt, so lange, bis endlich die ganze Geschichte erzählt ist. Und dann, erst dann, kommen jene Leute auf ihre Rechnung, die sagen, sie würden sich nur noch Filme mit einem Happy End anschauen, das andere kennten sie schon vom Alltag. Oder die Geschichte vom Bookshop, den eine Witwe - vielleicht um

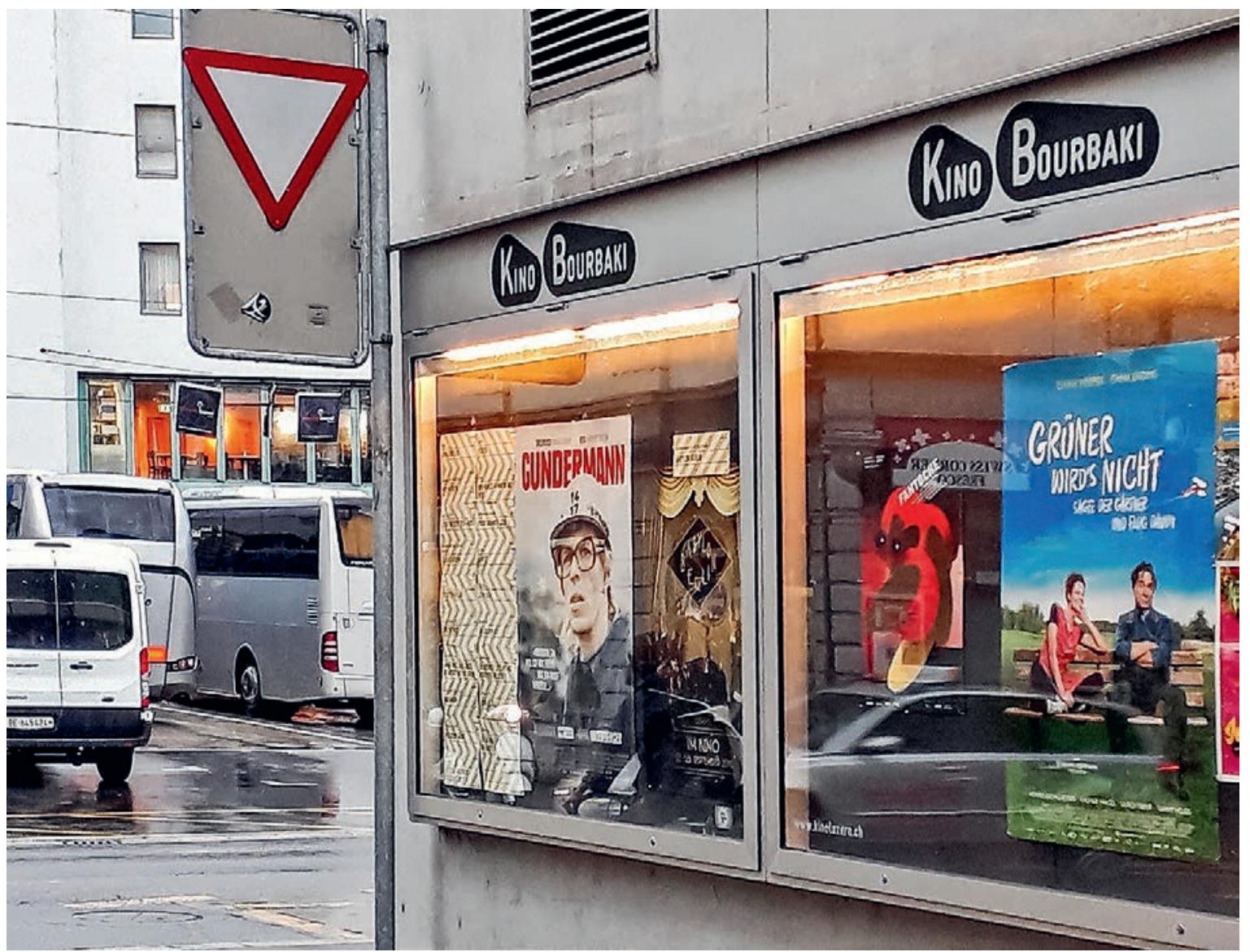

Welchen Film schauen wir uns heute im Kino Bourbaki an? 
die 50 - in einer kleinen Stadt am Meer aufmacht mit viel Liebe für das gute Buch (darunter zählt im Film auch Lolita von Vladimir Nabokov). Und allen Unkenrufen zum Trotz hat sie anfänglich grossen Zulauf bei den Arbeitereinwohnern, jedenfalls so lange, bis Neid (und Gier) dieses literarische Idyll zerstören. Vielleicht auch noch die Geschichte von «my lady», wie die Richterin in The Children Act genannt wird, eine Workaholic, die offensichtlich nicht realisiert, dass bei ihrer Berufsleidenschaft ihre Ehe zugrunde geht (nicht nur Ärzte haben Dienstwochenende!). Die Dialoge der beiden Partner sind Gesprächen im wirklichen Leben nachempfunden, sie tun weh und verletzen (das will man ja damit erreichen). Diese Filme sind stimmig: Es passt das wunderschöne Bild zu den Grossaufnahmen der Gesichter, es passt der ruhige Kameraschwenk, es passt der treffende Satzton, es passt die Geschichte, man glaubt sie. Es ist vielleicht fast etwas optischer Hochglanz, aber die Empathie des Regisseurs für seine Erzählung ist förmlich zu spüren. Und die Musik trägt die Geschichte, ob sie nun traurig, fröhlich oder am Schluss erlösend ist. Es ist die Stimmung, die visuell und akustisch überzeugt - es ist das stimmige Bild! Es muss nicht das Bild auf der Leinwand sein. Es kann auch die Beschreibung des Bildes im Buch sein, das sich dann im Kopf des Lesers festsetzt. Das kann so weit gehen, dass man nicht nur meint, das Bild zu sehen, sondern auch vermeint, die atmosphärischen Umstände des Bildes zu riechen: Wenn man zum Beispiel die Strasse nach Paris olfaktorisch wahrnimmt, gesäumt von einer braunen Kloake, die Zeugnis von der menschlichen Verdauung ablegt. Und wenn man Ammoniak in der Nase hat und begreift, wieso die Schweizer Milchkühe von Prinzessin Elisabeth, der Schwester von Louis XVI (das ist der, dessen Kopf die französische Monarchie beendet hat), nicht mehr in der Nähe des Schlosses von Versailles grasen möchten, weil die Ausdünstungen dieses "gigantischen Scheisshauses» eine nasale Beleidigung sind. Selten ist ein geschichtlich belegtes Märchen so real beschrieben worden wie im neuen Buch von Alex Capus [1] - es ist das stimmige Bild!

Und so geht diese Beschreibung des stimmigen Bildes vom Versailles des 18. Jahrhunderts über die kleinen Filme im Bourbaki (Kinohaus in Luzern) in die Versicherungsmedizin über: Denn Felix Bonnaire schreibt: «Die Begutachtung von Rotatorenmanschettenrupturen ist nur selten eine ganz eindeutige und klare Angelegenheit, weil sie viele Mosaiksteine in der Beweisführung der gutachterlichen Stellungnahme zusammentragen muss und nicht immer ein stimmiges Bild entstehen will» [2]. Aber - will man als (Versicherungs-)Mediziner die Natur der vorliegenden Lä- sion einem akuten Trauma oder einem degenerativen Prozess zuordnen, so müssen diese Mosaiksteine von Anamnese/Unfallmechanismus, zeitnahem klinischem Bild, Bildgebung und - falls operiert - intraoperativem situs so zusammenpassen, dass es dem Juristen möglich wird, das medizinische Bild in seinen juristischen Kontext zu stellen. Diese medizinische Seite setzt nicht nur die Kenntnis der Operation/Behandlung (und der entsprechenden Indikation) voraus, sondern auch das - wenn möglich - persönliche Lesen der vorliegenden Bildgebung und das Wissen um die entsprechende Fachliteratur. Aus all diesen Puzzleteilchen ergibt sich dann die möglichst umfassende Analyse, die - dann erst - eine Beurteilung der strittigen Lage erlaubt. Dabei muss die persönliche Empathie, die im klinischen Alltag einem Mediziner nicht abträglich, ja vielleicht gar zuträglich ist, ausgeschlossen werden. Das ist angesichts der zum Teil haarsträubenden Aussagen und Argumente in den Dokumenten nicht immer ganz einfach! Gefragt ist hier eine gnadenlose Sachlichkeit bar jeder persönlichen Emotion, gesucht ist die nachvollziehbare und vor allem überzeugende Schlüssigkeit.

Es ist aber auch so eine Sache mit dieser (für mich) intellektuellen Herausforderung in der Versicherungsmedizin. Angesichts der erforderlichen persönlichen Erfahrung erstaunt es, wie viele junge Leute die versicherungsmedizinischen Kurse besuchen und ein entsprechendes Zertifikat dann ihr Eigen nennen dürfen. Es mutet eigentlich auch eigenartig an, dass die medizinische Tätigkeit, die dazu angelegt ist, dem Kranken und Verletzten zu helfen, in einem Rechtsstreit mündet, ob die Behandlung nun lege artis vorgenommen wurde (und das ist sie leider nicht immer), und ob nun die Krankenkasse oder die Unfallversicherung für die Kosten zuständig sei. Das sind im Grunde enorm kostspielige "Auswüchse» einer an sich schon defizitären Medizin, die es ganzen Versicherungszweigen erlauben, davon gut zu leben. Aber trotz diesen Einwänden stellt die Versicherungsmedizin eine äusserst spannende Tätigkeit dar. Eine höchst fordernde Tätigkeit auch, weil sie verlangt, dass die nüchterne Darstellung und Beurteilung der Sachlage am Schluss in einem stimmigen Bild (ohne Belletristik) endet. Es ist das stimmige Bild, das gesucht wird - in der Kultur und in der Versicherungsmedizin.

\section{Bildnachweis}

Foto: Dominik Heim

\section{Literatur}

1 Capus Alex. Die Königskinder. Hanser Verlag 2018.

2 Bonnaire Felix. Begutachtung der Rotatorenmanschettenläsion. Trauma Berufskrankh. 2008;10(Suppl 1):16-24. 